\title{
Microbial community in high arsenic groundwater aquifers from Hetao Plain of Inner Mongolia, China
}

\author{
Y.H. Wang, P. Li, Z. Jiang, H. Liu, D.Z. Wei \& H.L. Wang \\ State Key Laboratory of Biogeology and Environmental Geology, China University of Geosciences, \\ Wuhan, P. R. China
}

\begin{abstract}
The microbial community composition in high arsenic groundwater aquifers was investigated by 454 pyrosequencing and illumina MiSeq sequencing approaches. Both groundwater and sediment samples were divided into low and high arsenic groups according to principal component analysis and hierarchical clustering. Microbial communities were significantly different among samples with different geochemistry. Predominant populations were Acinetobacter, Pseudomonas, Psychrobacter and Alishewanella in high reducing area, Alishewanella, Psychrobacter, Methylotenera and Crenothrix in agricultural area, and Thiobacillus, Pseudomonas and Hydrogenophaga in high arsenic sediments. Arsenic, $\mathrm{TOC}, \mathrm{NH}_{4}^{+}, \mathrm{Fe}(\mathrm{II})$ and $\mathrm{SO}_{4}^{2-}$ were important factors shaping indigenous microbial communities.
\end{abstract}

\section{INTRODUCTION}

Arsenic (As) contamination in groundwater aquifers is a serious environmental issue in many countries. Results of previous studies showed that As release and mobilization were usually controlled by a series of microbially mediated reactions and geochemical processes. However, microbial communities in groundwater and sediments with different geochemistry have yet to be fully understood.

\section{METHODS/EXPERIMENTAL}

\subsection{Sample collection and geochemical analysis}

Hangjinhouqi County is one of the most serious high arsenic groundwater threatened area located in the west of Hetao Plain. Groundwater samples from high reducing area and along agricultural drainage channels and sediments from three boreholes in Hangjinhouqi County were collected. Microbial samples were collected by filtering of $15 \mathrm{~L}$ fresh waters through $0.2 \mu \mathrm{m}$ filters. Geochemical parameters were measured in situ or sampled according to our previous studies (Wang et al., 2016).

\subsection{4 pyrosequencing and illumina MiSeq sequencing}

Pyrosequencing of the V4 region of the 16S rRNA gene was carried out from the 515F-end of the amplicons with Roche (454) genome sequencer FLX+ system (454 Life Sciences, USA) at SeqWright Inc (Houston, USA). Illumina sequencing were conducted by an
Illumina MiSeq 2000 instrument at the Yale Center for Genome Analysis. All raw data analysis was performed using the QIIME software package, version 1.5.0.

\subsection{Statistical analysis}

All statistical analysis in this study was performed, based on genus-level OTUs at the $97 \%$ similarity level under the package of Vegan in R.

\section{RESULTS AND DISCUSSION}

\subsection{Geochemistry}

Samples with different geochemistry presented distinct biogeochemical characteristics and redox properties. Groundwater and sediment samples could be divided into well-defined high and low As groups by Hierarchical clustering and PCoA analyses of geochemical parameters. Arsenic concentrations of groundwater samples along agricultural drainage channels showed positive correlations with $\mathrm{NH}_{4}^{+}$and TOC, while showed no obvious correlations with $\mathrm{Fe}(\mathrm{II})$ and $\mathrm{SO}_{4}^{2-}$, differencing from those samples from strongly reducing areas which were characterized with low concentrations of $\mathrm{SO}_{4}^{2-}$, negative ORP and high ratios of $\mathrm{Fe}(\mathrm{II})$ to $\mathrm{Fe}(\mathrm{III})$. In contrast, high As sediments in this study were under weak reducing conditions where As (III)/As (V) increased with depth from 0.02 to 0.52 and $\mathrm{SO}_{4}^{2-}$ /Total $\mathrm{S}$ ranged between 0.001 and 0.75 .

\subsection{Microbial richness and diversity}

The microbial community diversities of groundwater from highly reducing area and sediments were 
analyzed by 454 pyrosequencing. A total of 233,704 reads were obtained for 39 samples after removal of low-quality and chimeric sequences. A variety of taxa were observed at the $97 \%$ OTU level, with 12 267 observed and 23-355 predicted OTUs (based on Chao1) and coverage values ranging from $31.3 \%$ to $81.6 \%$. Richness and diversity indexes of these samples showed no significant correlations with the concentrations of As or any other geochemical variables. Illumina sequencing was used to examine the microbial communities of groundwater from agricultural drainage channels. Sequencing results revealed that a total of 329-2823 OTUs were observed at the 97\% OTU level. Microbial richness and diversity of high arsenic groundwater samples along the drainage channels were lower than those of low arsenic groundwater samples, but higher than those of high arsenic groundwaters from strongly reducing areas, implying that irrigation and drainage activities might affect local geochemical environment and thus enhance the diversity of microorganisms.

\subsection{Microbial compositions in high As groundwater aquifers}

Microbial community compositions at phylum level showed no distinct difference between high and low As groundwater samples as well as sediment samples. For groundwater samples from highly reducing areas and sediment samples, Acinetobacter, Psychrobacter and Alishewanella were more abundant in groundwater than sediments. The average abundances of Thiobacillus, Pseudomonas, Hydrogenophaga, Enterobacteriaceae, Sulfuricurvum and Arthrobacter were higher in sediments than groundwater samples. It was worth noting there were two populations Acinetobacter and Thiobacillus which highly contributed to the dissimilarities of these two kinds of samples (average abundance $30.45 \%$ and $12.31 \%$, respectively). Acinetobacter was distinctly dominated in groundwater with the markedly high relative abundance $(62.41 \%)$, and Thiobacillus was significantly abundant (average abundance $24.62 \%$ ) in sediments, while being absent in most of the groundwater (average abundance $0.01 \%$ ). The archaeal abundance was mostly lower than $1 \%$. Methanogens were distinctly predominant in most of the high arsenic groundwater, while similar results haven't been found in high arsenic sediments.

Illumina results of groundwater samples from agricultural drainage channels indicated that the bacterial community was composed of 62 phyla. Of these, only 9 phyla dominated each community, among which Proteobacteria was the most dominant group (32.02-86.50\%), followed by Firmicutes (0.16$18.48 \%)$, Actinobacteria (0.34-12.08\%) and Nitrospirae $(0-22.64 \%)$. Archaeal populations accounted only for $0.01-8.69 \%$ of the microbial populations. All detected archaeal populations belonged to the phyla Euryarchaeota and Crenarchaeota which represented by the classes of Parvarchaea, Methanomicrobia and Methanobacteria. These methanogenic populations might provide even stronger reducing conditions and thereby accelerate As release in groundwater aquifers in the Hetao Plain. However, the irrigation and drainage activities in agricultural area could bring more oxygen and the oxidizing conditions might weaken the role of methanogenic populations. At the genus level, Alishewanella, Psychrobacter, Pseudomonadaceae, Methylotenera, Crenothrix and Comamonadaceae were dominant communities in high As groundwater samples from agricultural drainage channels with high average abundances. These dominant populations indicated that As release in groundwater along agricultural drainage channels was correlated with microbial carbon, nitrogen and iron reactions. Different from samples from the highly reducing area, many oxidizing microbial populations such as Methylotenera and Crenothrix were found in the present study, indicating the possible influence of geochemical difference on microbial composition.

\subsection{Microbial community structure in relation to geochemistry}

Co-inertia analysis was carried out in order to reveal the relative importance of geochemical vectors that affect microbial community structures. Results revealed that geochemical variables had a significant influence on their microbial community composition. Arsenic is one of the key environmental factors that contribute to the difference in the geochemistry and microbial community structure in both groundwater and sediments. Besides, geochemical parameters including $\mathrm{As}, \mathrm{TOC}, \mathrm{SO}_{4}^{2-}, \mathrm{SO}_{4}^{2-} / \mathrm{TS}$ and $\mathrm{Fe}^{2+}$ were important factors causing the difference of the microbial community in groundwater samples from highly reducing area and sediment samples. Geochemical variables such as As, TOC, $\mathrm{NH}_{4}^{+}, \mathrm{Fe}$ and ORP might also affect the microbial composition from agricultural drainage channels. These important geochemical factors shaping the microbial community structure were different from those groundwater samples of highly reducing area and sediment samples in the Hetao Plain (Li et al., 2015).

\section{ACKNOWLEDGEMENTS}

This study was funded by National Natural Science Foundation of China (No. 41372348, 41521001).

\section{REFERENCES}

Li, P., Wang, Y., Dai, X., Zhang, R., Jiang, Z., Jiang, D., Wang, S., Jiang, H., Wang, Y. \& Dong, H. 2015. Microbial community in high arsenic shallow groundwater aquifers in Hetao Basin of Inner Mongolia, China. PloS one 10(5): e0125844.

Wang, Y., Li, P., Jiang, Z., Sinkkonen, A., Wang, S., Tu, J., Wei, D., Dong, H. \& Wang, Y. 2016. Microbial community of high arsenic groundwater in agricultural irrigation area of Hetao Plain, Inner Mongolia. Front. Microbiol. 7: 1917. 


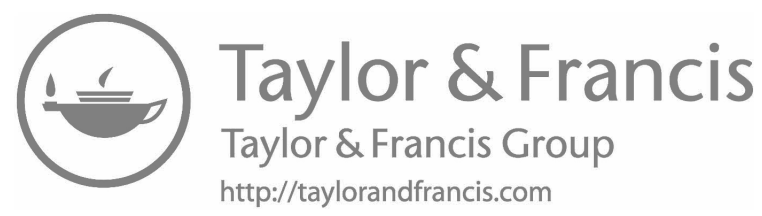


1.5 Spatial and temporal evolution of arsenic in mine waste and tailings 


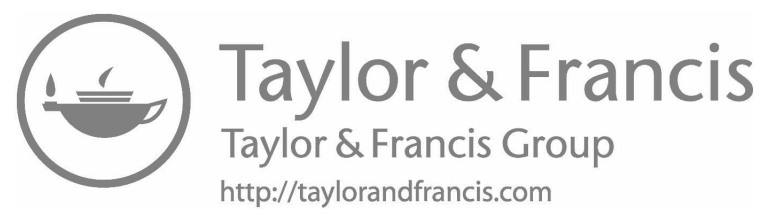

